\title{
Measurement of the electrical properties of a thundercloud through muon imaging by the GRAPES-3 experiment.
}

\author{
B. Hariharan ${ }^{* 1,2}$, S. Ahmad ${ }^{1,3}$, A. Chandra ${ }^{1,3}$, S.R. Dugad ${ }^{1,2}$, S.K. Gupta ${ }^{1,2}$, \\ Y. Hayashi ${ }^{1,4}$, P. Jagadeesan ${ }^{1,2}$, A. Jain ${ }^{1,2}$, S. Kawakami ${ }^{1,4}$, H. Kojima ${ }^{1,5}$, \\ P.K. Mohanty ${ }^{1,2}$, S.D. Morris ${ }^{1,2}$, Y. Muraki ${ }^{1,6}$, P.K. Nayak ${ }^{1,2}$, A. Oshima ${ }^{1,5}$, \\ P.S. Rakshe ${ }^{1,2}$, K. Ramesh ${ }^{1,2}$, B.S. Rao ${ }^{1,2}$, L.V. Reddy ${ }^{1,2}$, S. Shibata ${ }^{1,5}$, K. Tanaka ${ }^{1,7}$, \\ M. Zuberi ${ }^{1,2}$ \\ ${ }^{1}$ The GRAPES-3 Experiment, Cosmic Ray Laboratory, Raj Bhavan, Ooty 643001, India \\ ${ }^{2}$ Tata Institute of Fundamental Research, Mumbai 400005, India \\ ${ }^{3}$ Aligarh Muslim University, Aligarh 202002, India \\ ${ }^{4}$ Graduate School of Science, Osaka City University, Osaka 558-8585, Japan \\ ${ }^{5}$ College of Engineering, Chubu University, Kasugai, Aichi 487-8501, Japan \\ ${ }^{6}$ Institute for Space-Earth Environmental Research, Nagoya University, Nagoya 464-8601, Japan \\ ${ }^{7}$ Graduate School of Information Sciences, Hiroshima City University, Hiroshima 731-3194, \\ Japan \\ E-Mail: 89hariharanegmail.com
}

\begin{abstract}
The GRAPES-3 muon telescope located in Ooty, India records rapid ( $\sim 10 \mathrm{~min})$ variations in the muon intensity during major thunderstorms. Out of a total of 184 thunderstorms recorded during the interval April 2011-December 2014, the one on 1 December 2014 produced a massive potential of $1.3 \mathrm{GV}$. The electric field measured by four well-separated (up to $6 \mathrm{~km}$ ) monitors on the ground was used to help estimate some of the properties of this thundercloud including its altitude and area that were found to be $11.4 \mathrm{~km}$ above mean sea level (amsl) and $\geq 380 \mathrm{~km}^{2}$, respectively. A charging time of 6 min to reach $1.3 \mathrm{GV}$ implied the delivery of a power of $\geq 2 \mathrm{GW}$ by this thundercloud that was moving at a speed of $\sim 60 \mathrm{~km} \mathrm{~h}^{-1}$. This work possibly provides the first direct evidence for the generation of $\mathrm{GV}$ potentials in thunderclouds that could also possibly explain the production of highest energy $(100 \mathrm{MeV}) \gamma$-rays in the terrestrial $\gamma$-ray flashes.
\end{abstract}

36th International Cosmic Ray Conference -ICRC2019-

July 24th - August 1st, 2019

Madison, WI, U.S.A.

${ }^{*}$ Speaker. 


\section{Introduction}

Thunderstorms are a spectacular manifestation of discharge of massive electric potentials develop in thunderclouds during severe weather conditions. The first authoritative study of thunderstorms by Franklin dates back to 1750s [1]. A major advance in their understanding occurred in 1920s when the dipole structure was identified [2]. However, actual structure is more complex. The separation of electric charges in thunderclouds occurs when supercooled water-droplets make grazing contact with hail-pellets (graupel) polarized by the fine-weather electric field $\left(120 \mathrm{~V} \mathrm{~m}^{-1}\right)$ on Earth's surface. The rebounding droplets acquire positive charge and are carried by convective updraft toward the cloud-top while negatively charged graupel fall toward cloud-base due to gravity. This creates a vertical field that increases polarizing charge on graupel thus, accelerating this process and reinforcing vertical field, that grows exponentially until air insulation breaks down and triggers a lightning discharge. Graupel falling at a speed of $30 \mathrm{~mm} \mathrm{~h}^{-1}$ can produce vertical electric fields of $5000 \mathrm{~V} \mathrm{~cm}^{-1}$ in about 10 minutes involving the separation of $\sim 50 \mathrm{C}$ of charge, enough to initiate another lightning flash. The graupel movement sustains the process of producing a succession of lightning flashes at $\sim 30 \mathrm{~s}$ intervals [3]. Since the thickness of thunderclouds extends to several kilometers, potentials of $\geq 1 \mathrm{GV}$ could be generated [2].

A unique signature of massive electric potentials generated in thunderclouds was the discovery of terrestrial $\gamma$-ray flashes (TGFs) with MeV photons by the BATSE instrument aboard Compton

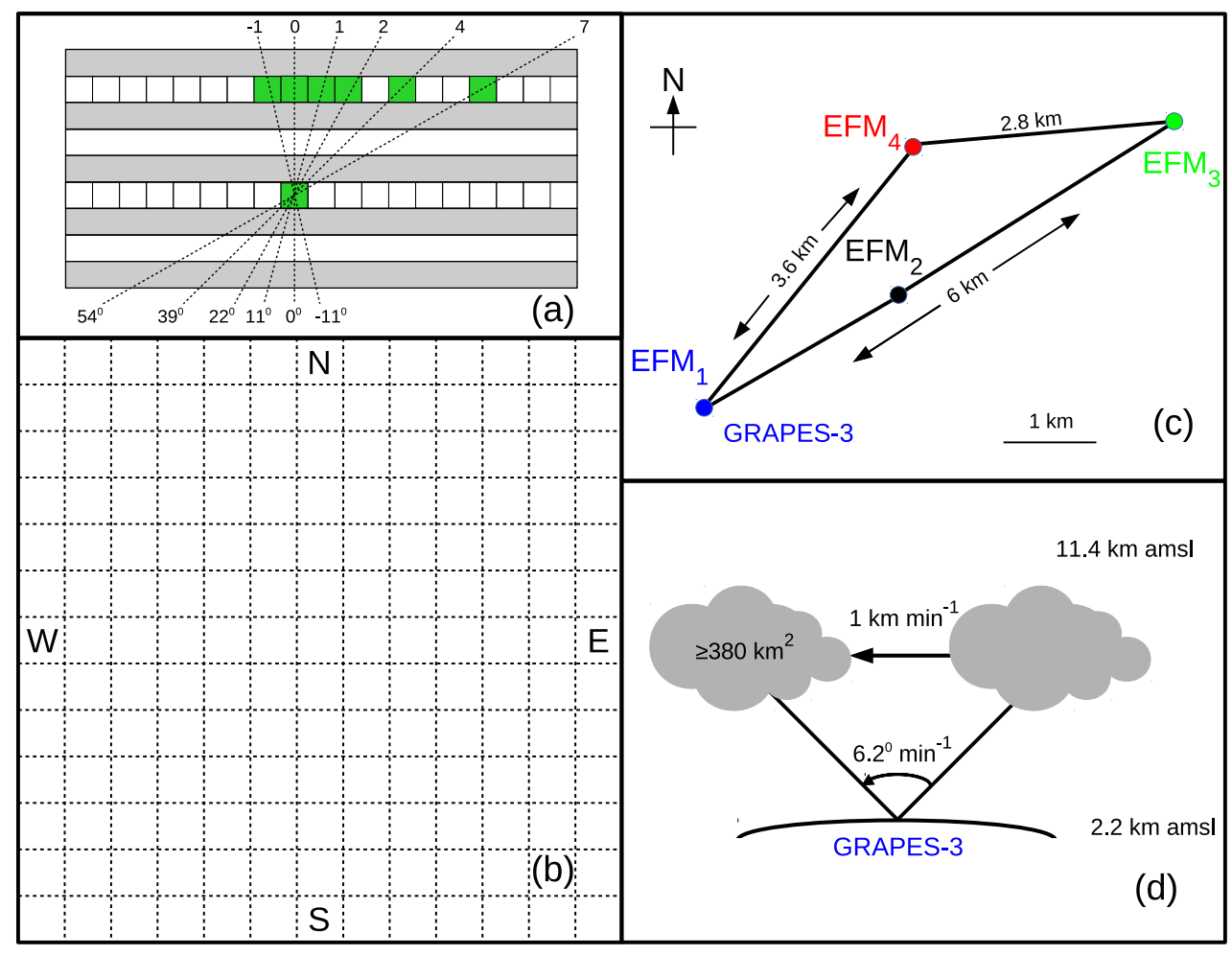

Figure 1: (a) Muon directions in a single projection plane from PRC geometry, (b) telescopic field of view (FOV) of 2.3 sr segmented into $13 \times 13=169$ directions, (c) locations of EFMs labeled 1 to 4, with maximum distance between the station to be $6 \mathrm{~km}$, (d) schematic of thundercloud movement describing linear and angular velocities, altitude and area. 
$\gamma$-ray observatory. The source of TGFs was identified to be thunderstorms in the lower tropical atmosphere [4]. Subsequently, the RHESSI data showed that $\gamma$-ray energies extended to $20 \mathrm{MeV}$, produced through bremsstrahlung of electrons of higher energies [5]. The results from the $\gamma$-ray burst monitor aboard Fermi satellite extended the maximum $\gamma$-ray energy to $40 \mathrm{MeV}$, implying the presence of $>100 \mathrm{MeV}$ electrons [6]. The detection of $\gamma$-ray energy of $100 \mathrm{MeV}$ by the AGILE satellite would however, require bremsstrahlung of very high-energy electrons and presence of potentials of hundreds of MV [7]. The maximum thunderstorm potential measured in balloon soundings is only $0.13 \mathrm{GV}$ [8], well short of the magnitude needed to produce $100 \mathrm{MeV} \gamma$-rays [7] and of $1 \mathrm{GV}$ predicted by Wilson [2]. MeV $\gamma$-rays produced in thunderstorms have been detected on the ground, both through triggered and natural lightening discharges, showing a close connection of the TGFs detected from space and from ground $[9,10]$. Early studies of the changes in muon intensity $\left(\mathrm{I}_{\mu}\right)$ at low-energies $(90 \mathrm{MeV})$ were shown to be correlated with the electric field of thunderstorms $[11,12]$ and confirmed by the results from Mt. Norikura [13] and elsewhere [14].

\section{The GRAPES-3 Muon Telescope}

The GRAPES-3 muon telescope (G3MT) in Ooty $\left(11.4^{\circ} \mathrm{N}, 2200 \mathrm{~m}\right.$ amsl) studies astrophysics of cosmic rays (CRs) through the measurement of $\mathrm{I}_{\mu}$. Its detection element is a proportional counter (PRC) made from steel pipes $(6 \mathrm{~m} \times 0.1 \mathrm{~m} \times 0.1 \mathrm{~m})$. The G3MT consists of 4 PRC layers under a $2 \mathrm{~m}$ thick concrete-roof, resulting in a threshold of $\mathrm{E}_{\mu}=1 \sec (\theta) \mathrm{GeV}$, for muons of zenith angle $\theta$. This 4-layer configuration enables muon reconstruction in two mutually perpendicular planes and the two PRC layers in same projection plane separated by $\sim 50 \mathrm{~cm}$ permit muon direction to be measured with $\sim 4^{\circ}$ accuracy as shown in Fig. 1a. Thus, the G3MT measures $I_{\mu}$ in 169 directions over a field of view (FOV) of $2.3 \mathrm{sr}$ as shown in Fig. 1b. Although, the solid angle of 169 directions differ significantly, but the area of thundercloud covered varies by only $19 \%$ [15]. Since $\sim 2.5 \times 10^{6}$ muons are recorded every minute, $\mathrm{I}_{\mu}$ gets measured to $0.1 \%$ precision $[16,17]$.

During thunderstorms, G3MT detects rapid changes ( $\sim 10 \mathrm{~min})$ in $\mathrm{I}_{\mu}$. Since the muon energies exceed $1 \mathrm{GeV}$, the presence of large electric potentials is implied. To probe this phenomenon, electric field monitors, hereafter "EFM" (Boltek model EFM-100 [18]) were installed in April
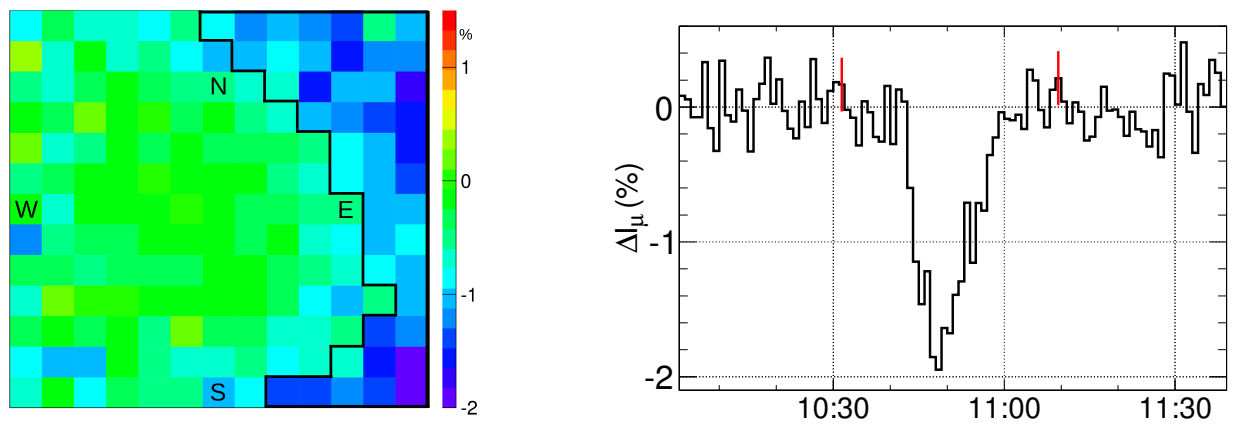

Figure 2: $\Delta \mathrm{I}_{\mu}$ during 18 min thunderstorm. 45 Figure 3: Maximum $\Delta \mathrm{I}_{\mu}=-2 \%$, starting out of 169, thunderstorm affected contiguous di- 10:42 UT, lasting $18 \mathrm{~min}$ seen during thunderrections are enclosed by dark boundary. Thun- storm of 1 December 2014. Vertical bars repdercloud angular size in $\mathrm{N}-\mathrm{S}=74.6^{\circ}$. resent $\pm 1 \sigma$ errors. 
2011 at four locations, at GRAPES-3, and three others a few km away as shown in Fig. 1c. The data collected during April 2011-December 2014 showed that 184 thunderstorms were detected both by G3MT and EFMs. The seven largest events with muon intensity variation $\Delta \mathrm{I}_{\mu} \geq 0.4 \%$ were shortlisted. However, except for the event on 1 December 2014 discussed here, the EFM profiles of remaining six events were extremely complex, that made association of $\Delta \mathrm{I}_{\mu}$ and electric field of a specific thundercloud difficult.

\section{Monte Carlo Simulations}

Thunderclouds are known to have a complex multipolar structure [3], but here it is assumed to be dipolar since the implications of such a structure can be easily simulated and a quantitative comparison of simulation output with experimental data could be used to obtain the average properties of the thundercloud by treating it as a parallel plate capacitor that can only provide an approximate estimate of its properties. To simulate muon response to thundercloud potential $\mathbf{V}$, a uniform vertical electric field $\mathbf{E}_{i}$ for the following three cloud thicknesses $\mathbf{D}_{i}$ were investigated, where $\mathbf{V}=\mathbf{E}_{i} \mathbf{D}_{i}$. (1) $\mathbf{D}_{1}=2 \mathrm{~km}$ for field between 8 and $10 \mathrm{~km}$ amsl, (2) $\mathbf{D}_{2}=7.8 \mathrm{~km}$ for field between the ground and $10 \mathrm{~km}$ amsl, (3) $\mathbf{D}_{3}=10 \mathrm{~km}$ for field between 10 and $20 \mathrm{~km}$ amsl. The dependence of $\Delta \mathrm{I}_{\mu}$ on $\mathbf{V}$ was obtained from Monte Carlo simulations, described in the next paragraph and was found to be same for cases (1) and (2). For case (3) $\Delta \mathrm{I}_{\mu}$ was $15 \%$ smaller than cases (1) and (2). The case (3) apart from being unrealistic, also required potentials higher than other two cases, thus, a uniform electric field applied between 8 and $10 \mathrm{~km}$ was used to provide a conservative estimate of the thundercloud potential $\mathbf{V}$.

The conversion of $\Delta \mathrm{I}_{\mu}$ into equivalent potential $\mathbf{V}$ is derived from simulations using the CORSIKA [19], that in turn relies on the choice of hadronic interaction generators. Here, FLUKA [20] and SIBYLL [21] were used for low- $(<80 \mathrm{GeV})$ and high-energy $(>80 \mathrm{GeV})$ interactions, respectively. When two other popular high-energy generators, namely, QGSJet [22] or EPOS [23] were used, an identical dependence of $\Delta \mathrm{I}_{\mu}$ on $\mathbf{V}$ was obtained. This is because the affected muons are produced by low-energy $(<80 \mathrm{GeV}) \mathrm{CRs}$ where the high-energy generators are not used. But, when the other two low-energy generators, GHEISHA [24] or URQMD [25] were used significant differences were observed. Compared to FLUKA, V inferred for GHEISHA was on an average
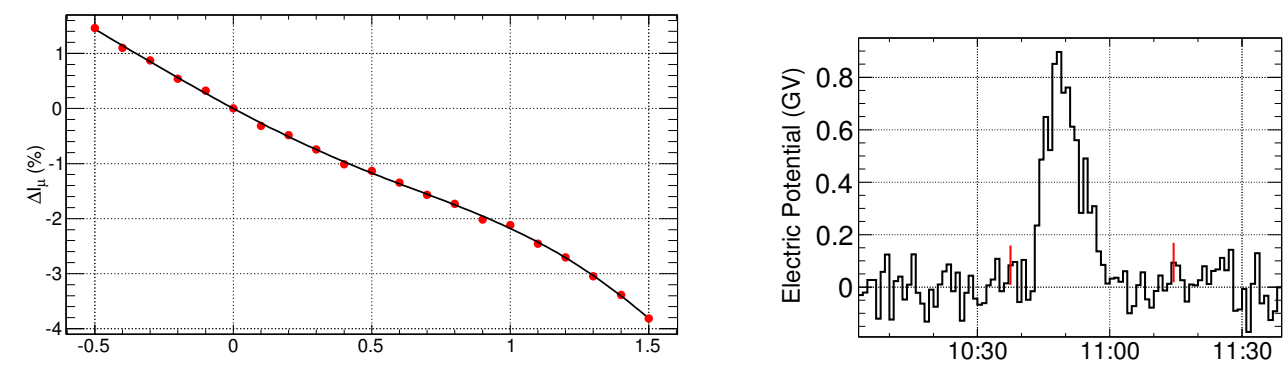

Figure 4: Dependence of $\Delta \mathrm{I}_{\mu}$ on electric po- Figure 5: Estimated electric potential shows a tential $(\mathrm{GV})$ across atmospheric layer $8-10 \mathrm{~km}$ maximum of $(0.90 \pm 0.08) \mathrm{GV}$ at $10: 48 \mathrm{UT}$ on 1 amsl, based on simulations for 45 directions December 2014. Vertical bars represent $\pm 1 \sigma$ ershown in Fig. 2. ror. 
15\% higher, and for URQMD 6\% higher. FLUKA was chosen as it provided the lowest and therefore, the most conservative estimate of the thundercloud potential. Next, the simulation of muons detected by the G3MT in each of the 169 directions were carried out, first with $\mathbf{V}=0$, and then by applying a $\mathbf{V}$ in the range $-3 \mathrm{GV}$ to $3 \mathrm{GV}$ in $0.1 \mathrm{GV}$ steps over a height from 8 to $10 \mathrm{~km}$ amsl as explained above. For each direction, the number of muons above the corresponding threshold energy were calculated. A high-statistics muon database of $10^{7}$ for $\mathbf{V}=0$, and $10^{6}$ muons for each non-zero $\mathbf{V}$ was created. This allowed the simulated $\Delta \mathrm{I}_{\mu}$ to be measured to $0.1 \%$ accuracy, much smaller than the error of $0.4-2.7 \%$ in real data.

The solar-wind introduces a diurnal variation in $\mathrm{I}_{\mu}$ that was removed by modeling with a higher-order polynomial after excluding thunderstorm affected 18 min data. The change in $\mathrm{I}_{\mu}$ during $18 \mathrm{~min}$ is shown in Fig. 2. A cluster of 45 contiguous directions enclosed by dark-boundary displays significant decrease in $\mathrm{I}_{\mu}$ as shown in Fig. 3. During 10:42-10:59 UT, a decrease of $2 \%$ is visible to the right of the dark boundary in Fig. 2 with a $20 \sigma$ significance.

Simulated dependence of $I_{\mu}$ for 45 directions on applied potential $\mathbf{V}$ is shown in Fig. 4. A positive $\mathbf{V}$ at thundercloud top relative to bottom would lead to energy-loss $\mathbf{e V}$ for $\mu^{+}$and gain $\mathbf{e V}$ for $\mu^{-}$. Since ratio $\mu^{+} / \mu^{-}>1.0$, the loss of detected $\mu^{+}$exceeds the gain of $\mu^{-}$. Thus, the sum of muons of both polarities decreases for positive $\mathbf{V}$ and beyond $1 \mathrm{GV}$ the slope gradually increases due to rapid increase in decay probability of $\mu^{+}$as seen in Fig. 4. This dependence is used to convert the measured $\Delta \mathrm{I}_{\mu}$ into equivalent $\mathbf{V}$ that peaks at $(0.90 \pm 0.08) \mathrm{GV}$ as shown in Fig. 5.

\section{Time Lapse of Cloud Movement}

The EFM records of electric field (sample rate $=20 \mathrm{~s}^{-1}$ ) show a smooth profile with an rms of $0.01 \mathrm{kV} \mathrm{m}^{-1}$ in all four cases, same as the EFM resolution, suggests the absence of major lightening. Hereafter, mean electric field $\left(\mathrm{min}^{-1}\right)$ is used for comparison with muon data $\left(\mathrm{min}^{-1}\right)$. Since all EFM profiles were similar and their amplitudes varied $22 \%$ around a mean $=3.3 \mathrm{kV} \mathrm{m}^{-1}$, they

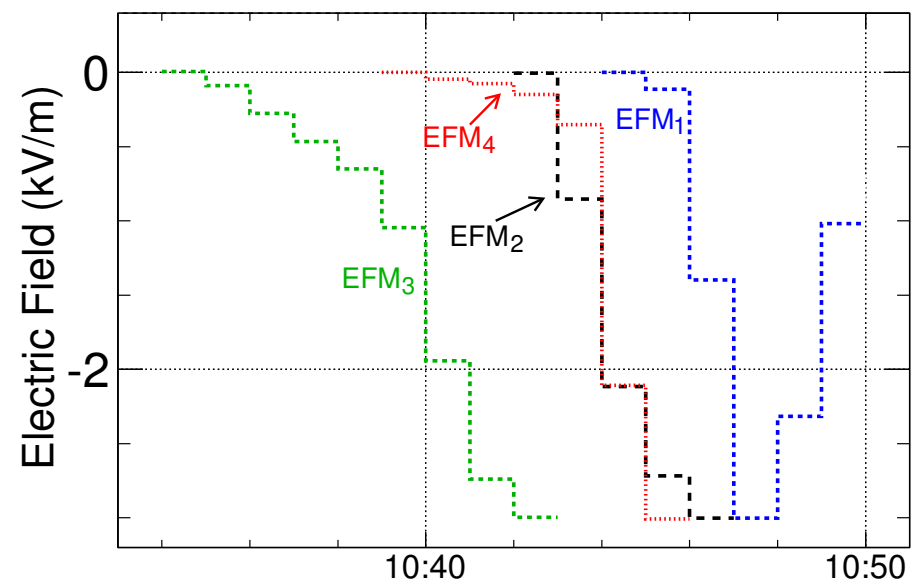

Figure 6: $\mathrm{EFM}_{3}$ profile appears first, followed by $\mathrm{EFM}_{2}$ and $\mathrm{EFM}_{4}$ after a 4 min delay. EFM ${ }_{1}$ comes last, 6 min after $\mathrm{EFM}_{3}$. Based on these EFM delays and locations from Fig. 1c, a thundercloud velocity of $1 \mathrm{~km} \mathrm{~min}^{-1}$ from east to west shown schematically in Fig. 1d is inferred. 


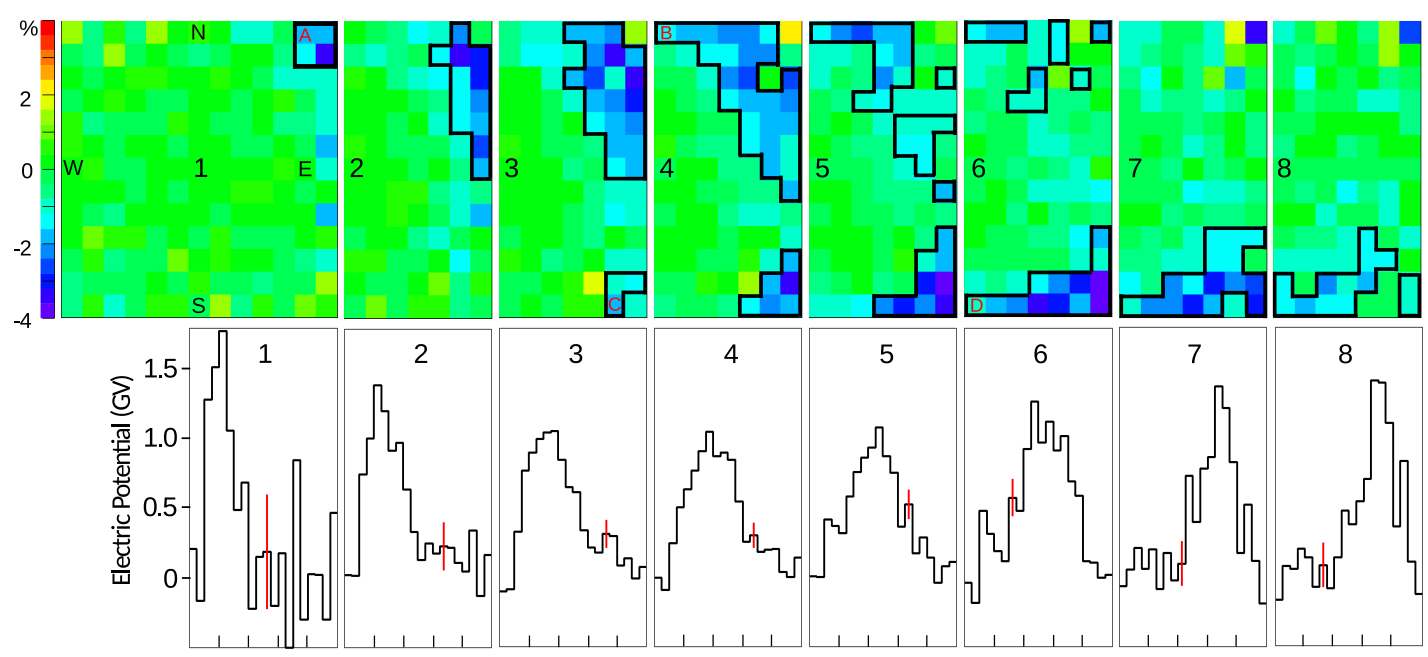

Figure 7: Top 8 panels show affected directions for successive 2 min exposures starting 1 December 2014 10:42 UT. Bottom 8 panels show estimated potentials needed to reproduce $\Delta \mathbf{I}_{\mu}$ shown in the corresponding panel above for a 20 min duration (10:41-11:00 UT). Maximum potentials of 1.8, 1.4, 1, 1, 1.1, 1.2, 1.3, $1.4 \mathrm{GV}$ (mean $=1.3 \mathrm{GV}$ ) observed for panels, 1 through 8 . Angular velocity of $6.2^{\circ} \mathrm{min}^{-1}$, inferred for directions (i) $\mathbf{A}$ to $\mathbf{B}$, (ii) $\mathbf{C}$ to $\mathbf{D}$ in north and south FOVs, respectively are shown in Fig. 1d. Vertical bar in each bottom panel corresponds to $\pm 1 \sigma$ error.

were normalized to $3 \mathrm{kV} \mathrm{m}^{-1}$ as shown in Fig. 6. $\mathrm{EFM}_{3}$ after a delay of 4 min was followed by $\mathrm{EFM}_{2}$ and $\mathrm{EFM}_{4}$, both of which overlapped. $\mathrm{EFM}_{1}$, closest to G3MT was delayed by 6 min relative to $\mathrm{EFM}_{3}$, indicating a thundercloud velocity of $\sim 1 \mathrm{~km} \mathrm{~min}^{-1}$, moving from $\mathrm{EFM}_{3}$ toward $\mathrm{EFM}_{1}$ as shown schematically in Fig. $1 \mathrm{~d}$.

Thundercloud movement in FOV may be studied by the displacement of its muon image in short 2 min exposures of $\Delta \mathrm{I}_{\mu}$. Because short exposures reduce muon statistics thus, regions that showed ( $\mathrm{I}_{\mu}$ ) decrease, (a) in contiguous directions or, (b) isolated directions over $\geq 2$ successive exposures were selected. In Fig. 7, $\Delta \mathrm{I}_{\mu}$ for first exposure starting 10:42 UT is shown for full FOV in first top-panel labeled $\mathbf{1}$. A decrease in 4 directions enclosed by dark-boundary is visible, the potential needed is shown in bottom-panel $\mathbf{1}$ of Fig. 7 that shows a maximum $\mathbf{V}=1.8 \mathrm{GV}$ during 10:41-11:00 UT. From the second panel onward, only 91 affected directions in the east are displayed. In top-panel 2, 12 affected directions require a maximum $\mathbf{V}=1.4 \mathrm{GV}$. This decreases to $1 \mathrm{GV}$ for panels 3 (23) and 4 (32). Then it increases to $1.1 \mathrm{GV}$ and $1.2 \mathrm{GV}$ for panels 5 (28) and 6 (23), respectively. Finally, reaches $1.4 \mathrm{GV}$ for panels 7 (16) and 8 (13). Integer values in the parenthesis next to each panel-number indicate the number of affected directions, highlighted by dark-boundary in the corresponding top-panels.

Successive panels in Fig. 7 show the west boundary of the muon image moving from east-towest in north-FOV. For example, it moved from direction $\mathbf{A}$ in top-panel $\mathbf{1}$ to $\mathbf{B}$ in top-panel $\mathbf{4}$ in 6 min implying an angular velocity of $6.2^{\circ} \mathrm{min}^{-1}$ as depicted in Fig. $1 \mathrm{~d}$. A movement of $6.2^{\circ} \mathrm{min}^{-1}$ of the muon image is seen in south-FOV from $\mathbf{C}$ to $\mathbf{D}$ in top-panels $\mathbf{3}$ and $\mathbf{6}$, respectively. A similar movement is also reflected in the progressive shift of peak voltage in the eight bottom-panels of Fig. 7. The angular velocity is combined with linear velocity from EFMs, then a height of $11.4 \mathrm{~km}$ amsl is obtained, comparable to typical thundercloud height [3]. The measured velocity and height are consistent with subtropical jet stream in south India [26]. 


\section{Electrical Properties of the Cloud}

In north-south direction the muon image covers the full FOV that corresponds to an angular size of $74.6^{\circ}$ as seen in Fig. 2. This implies a radius of $\geq 11 \mathrm{~km}$, very similar to average thundercloud radius $(\sim 12 \mathrm{~km})$ [27] and yields total area of this thundercloud of $\geq 380 \mathrm{~km}^{2}$. A thundercloud with infinitesimally thin charged regions, separated by $2 \mathrm{~km}$ acts as a parallel-plate capacitor of capacitance $\geq 1.7 \mu \mathrm{F}$. But in reality thickness of charged regions is comparable to their separation that reduces capacitance by $\sim 50 \%$ to $\geq 0.85 \mu \mathrm{F}$. $\mathbf{V}=1.3 \mathrm{GV}$ would require total charge $\mathrm{Q}=\geq 1100$ Coulomb and energy of $\geq 720 \mathrm{GJ}$ stored in this thundercloud. A $1.3 \mathrm{GV}$ potential across the thundercloud with its two charged regions of thickness $2 \mathrm{~km}$ each and a distance of $2 \mathrm{~km}$ between them implies an average field of $2.2 \mathrm{kV} \mathrm{cm}^{-1}$ which is lower than the breakdown field at high altitudes [3]. The mean time to reach the maximum potential shown in eight bottom panels in Fig. 7 is 6 min. Thus, the thundercloud would have delivered a power of $\geq 2 \mathrm{GW}$, comparable to single biggest nuclear reactors [28], hydroelectric and thermal power generators [29]. Separation of $2 \mathrm{~km}$ used is reasonable since it extends the thundercloud top into tropopause that defines the limit of cumulonimbus clouds producing major thunderstorms in the atmosphere [3]. Since the capacitance, total charge, energy stored and power delivered by a thundercloud vary inversely with the separation of its charged layers, thus these parameters can be easily calculated for any other separation.

The potential can be measured by integrating electric field over thundercloud height. However, in general the field measured by instruments aboard aircraft and balloons span a region much smaller than the thundercloud height and therefore, can not provide a reliable estimate of the potential. On the other hand, the $\Delta \mathrm{I}_{\mu}$ depends on the thundercloud potential and is virtually independent of its electric field and/or height. This makes muon telescopes with $\mathrm{GeV}$ threshold such as the G3MT ideal for measuring GV potentials in thunderclouds. However, such high-potentials can not be indefinitely sustained and a breakdown of air would result in acceleration of electrons to $\mathrm{GeV}$ energies. It is conceivable that bremsstrahlung emission from $\mathrm{GeV}$ electrons could produce photons ranging from a few to beyond $100 \mathrm{MeV}$ in a short flash of terrestrial $\gamma$-rays.

\section{Conclusions}

The GRAPES-3 muon telescope is well-suited to measure the electric potential developed in thunderclouds as shown for the 1 December 2014 event where a peak electric potential of $1.3 \mathrm{GV}$ was measured. This value is an order of magnitude larger than the previously reported maximum of $0.13 \mathrm{GV}$. This possibly is the first direct evidence for the generation of GV potentials in thunderclouds, consistent with the prediction of C.T.R. Wilson, 90 years ago [2]. The existence of GV potentials could explain the production of highest energy $\gamma$-rays in terrestrial $\gamma$-ray flashes discovered 25 years back [4]. It is shown that a $\geq 2 \mathrm{GW}$ of power, comparable to single biggest nuclear reactors [28], hydroelectric and thermal power generators [29] was delivered by this thunderstorm that was estimated to be moving at speed of $60 \mathrm{~km} \mathrm{~h}^{-1}$ near the top of the troposphere. Despite a simplified structure of the thundercloud used here, the present work provides reasonable insights into the physical state of the thunderstorms [30]. 


\section{Acknowledgments}

D.B. Arjunan, V. Jeyakumar, S. Kingston, K. Manjunath, S. Murugapandian, S. Pandurangan, B. Rajesh, K. Ramadass, V. Santhoshkumar, M.S. Shareef, C. Shobana, R. Sureshkumar are thanked for assistance in running the GRAPES-3 experiment. We thank SERB-DST of government of India and ICRC organizers for providing partial support to attend this conference.

\section{References}

[1] B. Franklin, Experiments and Observations on Electricity made at Philadelphia in America (London, 1751); B. Franklin, Phil. Trans. 47, 565 (1752).

[2] C.T.R. Wilson, Nucl. J. Franklin Inst. 208, 1 (1929); Proc. Phys. Soc. Lond. 37, 32D (1924); Proc. R. Soc. Lond. A 236, 297 (1956).

[3] B.J. Mason, Proc. R. Soc. Lond. A 327, 433 (1972); ibid 415, 303 (1988); J. Mason and N. Mason, Eur. J. Phys. 24, S99 (2003); E.R. Williams, Sci. Am. 259, 88 (1988); C.P.R. Saunders, Space Sci. Rev. 137, 335 (2008).

[4] G.J. Fishman et. al, Science 264, 1313 (1994).

[5] D.M. Smith, L.I. Lopez, R.P. Lin and C.P. Barrington-Leigh, Science 307, 1085 (2005).

[6] M.S. Briggs et. al, J. Geophys. Res. 115, A07323 (2010).

[7] M. Tavani et. al, Phy. Rev. Lett. 106, 018501 (2011).

[8] T.C. Marshall and M. Stolzenburg, J. Geophys. Res. 106, 4757 (2001).

[9] J.R. Dwyer et. al, Science 299, 694 (2003).

[10] R. Ringuette et. al, J. Geophys. Res. 118, 7841 (2013).

[11] V.V. Alexeenko et al., Proc. 20th International Cosmic Ray Conf. 4, 272 (1987).

[12] L.I. Dorman et. al, J. Geophys. Res. 108, 1181 (2003).

[13] Y. Muraki et al. Phys. Rev. D 69, 123010 (2004).

[14] A. Chilingarian et al. Sci. Rep. 7, 1371 (2017).

[15] B. Hariharan et al. Proc. Sci. PoS (ICRC2017) 481.

[16] S.K. Gupta et al. Nucl. Instrum. Methods A 540, 311 (2005); Y. Hayashi et al. Nucl. Instrum. Methods A 545, 643 (2005).

[17] P.K. Mohanty et al. Phys. Rev. Lett. 117, 171101 (2016); P.K. Mohanty et al. Phys. Rev. D 97, 082001 (2018).

[18] https://www.boltek.com/EFM-100C_Manual_121415.pdf

[19] https://www.ikp.kit.edu/corsika

[20] http://www.fluka.org/references.html

[21] E.J. Ahn, R. Engel, T.K. Gaisser, P. Lipari, and T. Stanev, Phys. Rev. D 80, 094003 (2009).

[22] N.N. Kalmykov, S.S. Ostapchenko, and A.I. Pavlov, Nucl. Phys. B Proc. Suppl. 52B, 17 (1997).

[23] T. Pierog et al., arXiv:1306.0121 [hep-ph] (2013). 
[24] http://cds.cern.ch/record/162911/files/CM-P00055931.pdf

[25] http://urqmd.org

[26] https://www.britannica.com/science/subtropical-jet-stream

[27] http://www.nssl.noaa.gov/primer/tstorm/tst_basics.html

[28] I. Pioro and R. Duffey, ASME J. of Nucl. Rad. Sci. 1, 011001 (2015).

[29] https://www.power-technology.com/features/feature-the-10-biggest-hydroelectric-power-plants-inthe-world;

https://www.power-technology.com/features/feature-giga-projects-the-worlds-biggest-thermal-powerplants.

[30] B. Hariharan et al., Phys. Rev. Lett. 122, 105101 (2019). 